\title{
Fecal microbiota transplantation in recurrent Clostridium difficile infection: the first prospective study of 30 patients in Romania
}

\author{
Mihaela Laszlo $^{1 *}$, Oliviu Pascu ${ }^{2,3}$, Daniel-Corneliu Leucuta ${ }^{3}$, Vasile Andreica ${ }^{2,3}$ \\ 1. IRGH Octavian Fodor Cluj Napoca, Romania \\ 2. Regional Institute of Gastroenterology and Hepatology, Cluj-Napoca, Romania \\ 3. University of Medicine and Pharmacy "Iuliu Hatieganu”, Cluj-Napoca, Romania
}

\begin{abstract}
Introduction: The infection with Clostridium difficile has increased in incidence worldwide and it raises many problems with regard to therapy, resistance to treatment and especially recurrence. Recurrence is frequent in patients treated for Clostridium difficile infection, requiring vancomycin by mouth, with limited alternatives. The literature shows that one of the most efficient treatment methods in Clostridium difficile infection is the transplantation of gut microbiota, also known as fecal microbiota transplantation.

Aim: We present our results following FMT performed in patients with recurrent Clostridium difficile infection, and propose a simple and effective protocol for fecal microbiota transplantation.

Study design: The study was prospective. The phases of the FMT procedure: assessment of patient eligibility, patient's consent, identification and screening of donors, discontinuation of antibiotics (vancomycin, metronidazole) 3 days prior to the procedure.

Methods: Between 2013 and 2015, FMT was performed in 30 patients with recurrent Clostridium difficile infection, by direct infusion of extensively processed donor fecal matter via colonoscopy. We followed up the patients for 12 months.

Results: Immediate post-transplantation outcome in what concerns stool frequency during the follow-up period (7 days) was encouraging in 93.33\% of patients. The donors were healthy individuals (53\% 1st degree relatives), previously screened for possible infections and infestations. This result was sustained at 6-month and 12-month follow-up. Post-transplantation recurrence occurred in $6.67 \%$ (2 patients), which responded well to treatment and did not require a new vancomycin course.

Conclusions: Fecal microbiota transplantation via colonoscopy is effective, safe, easy to perform, it yields lasting results and is therefore a good option for recurrent or treatment-resistant Clostridium difficile infection.
\end{abstract}

Keywords: Clostridium difficile, recurrence, fecal transplantation, donor

Received: $1^{\text {st }}$ December 2017; Accepted: 14 $4^{\text {th }}$ March 2018; Published: $19^{\text {th }}$ March 2018

*Corresponding author: Laszlo Mihaela, IRGH Octavian Fodor Cluj Napoca, Romania

E-mail: miham83@yahoo.com 


\section{Introduction}

Clostridium difficile is a mobile, anaerobic gram-positive bacillus which is commonly present in the colon bacterial flora in more than $50 \%$ of children under the age of one, and this percentage reduces to about $3 \%$ in adults. About a quarter of the persons who had infections caused by Clostridium difficile species relapse, either because the initial infection was not completely healed, or because they come in contact with a new strain $[1,2,3]$.This actual epidemic is acknowledged by many countries, with reports from Europe [4], Taiwan [5] and Korea [6]. In Romania, the increase in Clostridium difficile infection (CDI) incidence and severity has been reported since the first months of 2011 - with the onset of the etiological investigation of CDI-compatible pathology [3].

For mild cases of $C D I$, supportive therapy, along with discontinuation of the antibiotic treatment that produced the infection and therapy with Metronidazole for 10-14 days, may be sufficient; moderate and persistent cases, however, require 10 to 14 days of oral metronidazole/ vancomycin or both.

Fidaxomycin is a new macrocyclic antibiotic, which selectively eradicates Clostridium difficile and was shown to have rates of clinical recovery comparable to vancomycin; however, it, too, is very expensive.

Despite these therapeutic options, the disease relapses in up to $5-35 \%$ of cases after initial therapy with metronidazole and vancomycin, and recurrences are more difficult to manage [7].

Possible alternatives and auxiliary options include fecal microbiota transplantation, intravenous immunoglobulin and monoclonal antibody therapy [8], but none have been studied in terms of efficacy and long-term side effects.

The fecal microbiota transplantation (FMT), also known as "fecal transplantation", is rapidly gaining in acceptance as a viable, safe and effec- tive treatment for recurrent Clostridium difficile infection.

FMT in $C D I$ theoretically works by replacing or consolidating the protective colonic microbiota, which was disrupted by antibiotics and/or iatrogenic factors [9]. Once disturbed, the normal intestinal flora loses its ability to self-protect becoming vulnerable, allowing germs such as Clostridium difficile to dominate. FMT recreates a balanced colonic microbiota, resisting colonisation and suppressing Clostridium difficile [10].

The first report on the use of FMT for the treatment of $C D I$ was published in 1983; a patient experienced prompt and complete remission of gastro-intestinal symptoms after receiving a FMT [11].

Although the impact of FMT on the immune system is complex and unpredictable, important research on the effect of these microbes on the host is under way.

FMT for recurrent $C D I$ is not yet the "standard/ acknowledged" therapy; nevertheless, its rate of success constantly exceeds $90 \%$ [12]; it is the adequate option for patients unable to clear the infection despite traditional management.

In this article, we aim to summarize the information on this therapeutic option, focusing on its methodology, in order to facilitate its application and acceptability.

\section{Material and methods}

\section{Patients}

FMT was performed in 30 patients between December 2014 - January 2016. Informed consent was obtained from all individual participants included in the study. All patients had experienced at least one recurrence of Clostridium difficile infection after standard treatment with metronidazole and vancomycin (10-14 days). We mention that out of the 30 patients enrolled in the study, one patient was minor. There were 
no patients who were intolerant to both therapies according to the protocol.

All patients signed an informed consent, which included a discussion on the standard colonoscopy, the risks of sedation, haemorrhage and perforation, as well as the infection and allergy risks. All possible reactions to FMT were discussed and documented.

The patients were instructed to discontinue the antibiotics 2 to 3 days before the procedure. The preparation for colonoscopy involved a 4000 macrogol solution (Fortrans) on the day preceding the FMT.

At 1 month, 6 and 12 months post FMT, patients were presented in outpatient clinic to for harvesting of toxin $\mathrm{A}$ and $\mathrm{B}$.

The participants were excluded from the analysis if their CD antibiotic therapy had been incomplete (less than $80 \%$ of the standard cure), the first episode of infection, or in case the A and B toxins were negative after antibiotic therapy and the patient was symptomatic. There were a total of 70 cases of Clostridium difficile in the period December 2014 - January 2016.

\section{Study design}

The study was approved by the Ethics Committee of "Octavian Fodor" Regional Institute of Gastroenterology and Hepatology, Cluj Napoca 17649/ 3 December 2014 and Ethics Committee of the University of Medicine and Pharmacy "Iuliu Hatieganu", Cluj-Napoca - 269/ 6 December 2014.

The phases of FMT procedure were: assessment of patient eligibility, patient's consent, identification and screening of donors, discontinuation of antibiotics (vancomycin, metronidazole) 3 days prior to the procedure.

Total hospitalization for FMT for each patient was approximately 17 days:

-the 7-day pre-screening period, reserved for: signing the informed consent, filling in the questionnaire of bowel movement assessment, according to the Bristol scale (number of bowel movements/day, consistency), applying the inclusion and exclusion criteria, lab tests (erythrocyte sedimentation rate -ESR, $\mathrm{C}$ reactive protein- CRP), identifying and collecting samples from donors.

- the fecal transplant, 3-5 days: pre-administration period (1-2 days) for collecting stool samples; administration of the fecal transplant ( 1 day) and the post administration period (1-2 days) for collecting stool samples, inflammatory tests (ESR, CRP) and monitoring clinical symptoms (fever, pain).

- follow-up period in hospital after FMT (3-5 days): questionnaire of bowel movement assessment, according to the Bristol scale (number of bowel movements/day, consistency), stool samples.

We used a questionnaire for the assessment of stool consistency and frequency, according to the Bristol scale, in the pre-screening and follow-up periods [15].

Another questionnaire was used for assessment of compliance with the antibiotic treatment for Clostridium difficile infection; it included information on the number of antibiotic courses administered until FMT, on whether other antibiotics were prescribed for another infection before identification of Clostridium difficile, and on adverse effects, both general (fever) and digestive (nausea, bloating, abdominal pain).

All patients were followed up for 12 months after FMT by telephone (every week of the first month after FMT, then every month for 11 months), and were re-admitted in case of complications.

\section{Donors}

The potential stool donors were identified by the subjects themselves, being relatives of grade I or II and in some patients being the wife/ husband. 
The donors were excluded if they had taken antibiotics 90 days prior to procedure or in case they did not meet the eligibility criteria: stool screening- ova and parasite exam, stool culture (usually includes: Salmonella, Shigella, Escherichia coli, O157:H7, Yersinia enterocolitica, and Campylobacter), Clostridium difficile toxins $A$ and $B$, rotavirus antigen; serology- HIV-1 and HIV-2, Hepatitis A, B and C; gastrointestinal comorbidities - history of inflammatory bowel disease, history of irritable bowel syndrome, chronic constipation or diarrhoea, history of gastrointestinal malignancies; other conditions - atopy, autoimmune conditions, such as multiple sclerosis, fibromyalgia, chronic fatigue syndrome.

\section{FMT procedure}

The fecal suspension $(\sim 150 \mathrm{ml})$ was diluted in $0.9 \%$ sterile saline solution up to a volume of 400-425 ml. Subsequently, the suspension was filtered several times. This suspension was poured into a sterile vial and administered within one hour. The administration method was transcolonic via colonoscopy to the ileum in $90 \%$ of patients and $10 \%$ to the level of the cecum, after standard preparation (Fortrans split - in doses that varied according to each patient's bowel transit).

The patients received $4 \mathrm{mg}$ of loperamide in order to reduce gut motility immediately after FMT and 6 hours after the procedure. Patients received a normal diet after about 5 hours. Serological tests were evaluated every day. The patients were discharged at 3 days after FMT.

Three days prior and after the procedure the patients no longer received vancomycin or metronidazole. For the following 7 days, the patients were asked to report any possible symptoms (fever, bloating, abdominal pain).

\section{Statistic analysis}

Categorical data was described as counts and percentages. Continuous normally distributed data were presented as means and standard deviations (SD), while data non following the normal distribution were presented as medians and interquartile ranges (IQR) and ranges. Confidence intervals of $95 \%$ were used for means and bootstrapped ones for medians. Normality of the data was assessed with quantile-quantile plots, stripcharts and Shapiro-Wilk tests. We used the Friedman test to compare repeated measurements of non-normally distributed data, the Kruskal-Wallis test (to compare multiple independent groups regarding non-normally distributed continuous variables) and the Fisher exact test (to assess the dependence between categorical variables). We considered two tailed $p$-values $<0.05$ to be statistically significant. R environment for statistical computing and graphics, version 3.2.3 was used for all statistical computations.

\section{Results}

We enrolled 30 patients (14 females, 16 males), aged between 5 and 83 years; all patients had had at least one relapse of Clostridium difficile infection, with a mean of 2 (table I, II). The duration of CDI infection until the FMT ranged between 4 and 8 months. Twenty-one patients underwent vancomycin and metronidazole therapy according to protocol (vancomycin $500 \mathrm{mg}$ every 6 hours and metronidazole $500 \mathrm{mg}$ every 8 hours by mouth for 14 days), repeated for each relapse, while the remaining 9 patients received either metronidazole (4 patients) or vancomycin (5 patients), dosed according to protocol. The 9 patients required a different therapy due to intolerance to one of the antibiotics. The treatment described has been done for previous recurrences and not for the current episode. The number of bowel movements ranged between 3 and 15/day, with a mean of 7.6.

A single patient had no associated conditions (a hospital worker); the others had various comorbidities associated with decreased immune 
Table I. Clinical and laboratory characteristics of patients

\begin{tabular}{lll}
\hline Characteristic & Mean (SD)/Median (IQR) & range \\
\hline Age, mean (SD) & $57.8(16.27)$ & $5-83$ \\
\hline $\begin{array}{l}\text { Nr bowel movements at 7 days before FMT, median } \\
\text { (IQR) }\end{array}$ & $7(6-10)$ & $3-15$ \\
\hline Nr relapses, mean (SD) & $2(0.91)$ & $1-4$ \\
\hline CRP (mg/l), median (IQR) & $3.58(1.21-16.5)$ & $0.4-28$ \\
\hline ERS (mm/h), median (IQR) & $35(20-56.75)$ & $6-105$ \\
\hline Hb (g/l), mean (SD) & $11.71(1.32)$ & $8-14.6$ \\
\hline Nr bowel movements at 7 days after FMT, mean (SD) & $2(1-3)$ & $1-4$ \\
\hline Toxin A and B after FMT, median (IQR) & $0.03(0.02-0.27)$ & $0-0.9$ \\
\hline CRP (mg/l) after FMT, median (IQR) & $1(0.7-7.5)$ & $0.4-20$ \\
\hline ESR (mm/h) after FMT, median (IQR) & $21(20-40)$ & $6-80$ \\
\hline Nr bowel movements at 6 months, median (IQR) & $1(1-2)$ & $1-3$ \\
\hline Nr bowel movements at 1 year, median (IQR) & $1.5(1-2)$ & $1-3$ \\
\hline
\end{tabular}

(SD - standard deviation, IQR - interquartile range, FMT-fecal microbiota transplantation, ESR - erythrocyte sedimentation rate, $\mathrm{CRP}-\mathrm{C}$ reactive protein, $\mathrm{Hb}$ - haemoglobin)

response or with requirement for antibiotic therapy (table II). The most used antibiotics were ceftriaxone, ciprofloxacin and amoxicillin-clavulanate. The mean number of relapses of CDI was 2.33 in patients treated with vancomycin and metronidazole, 1.5 in patients treated with vancomycin and 1 in those treated with metronidazole - a statistically significant difference between them (the metronidazol group being statistically significant, different than vancomycin and metronidazole group regarding the number of relapses, in the post-hoc pairwise comparisons $\mathrm{p}=0.004$ ) (tabel III).

All patients tolerated colonoscopy well. In 17 patients, the aspect of the colonic mucosa was normal; in 7 patients we found ulceration on certain segments of the colon, while in other 6 patients we found pseudomembranes.

After FMT, some patients developed certain clinical symptoms, as follows: fever (8 patients) in the first 3 days post FMT, which remitted spontaneously after the 3 days. Two patients experienced severe abdominal pain in the first 2 days post FMT, which responded to pain medication (tabel IV). The rest experienced moderate (10 patients) and mild pain (13 patients) that did not require medication.

Even though it is not an important breakthrough in view of the number of patients and the fact that it did not influence the results of the study, there was a slight increase in inflammatory markers relative to baseline levels.

Approximately 7 days after FMT 2 patients experienced a CDI relapse, with clinical symptoms and toxin A and B positive Clostridium strains; the remaining 28 patients did not experience any post FMT relapses, had no toxin positive strains and no symptoms in the following 12 months. The 2 relapsing patients had to undergo another vancomycin and metronidazole protocol for 14 days after FMT, followed by a relapse-free period during the rest of the 12 months of follow-up.

The post-FMT outcome in 28 patients was favourable (had no toxin positive strains and no symptoms), with a statistically significant im- 
Table II. Clinical characteristics pre transplantation

\begin{tabular}{|c|c|}
\hline Characteristic & Number $(\%)(n=30)$ \\
\hline Female & $14 / 30(46.67)$ \\
\hline Number relapses & $\begin{array}{l}1: 10 / 30(33.33) \\
2: 12 / 30(40) \\
3: 6 / 30(20) \\
4: 2 / 30(6.67)\end{array}$ \\
\hline Antibiotics for Clostridium difficile & $\begin{array}{l}\text { Metronidazole: } 5 / 30(16.67) \\
\text { Vancomycin: 4/30 (13.33) } \\
\text { Vancomycin and Metronidazole: } 21 / 30(70)\end{array}$ \\
\hline Colonoscopy & $\begin{array}{l}\text { normal: } 17 / 30(56.67) \\
\text { pseudomembranes: } 6 / 30(20) \\
\text { ulcerations: } 7 / 30(23.33)\end{array}$ \\
\hline Associated conditions & $\begin{array}{l}\text { Gastric adenocarcinoma, under chemotherapy: } 1 / 30(3.33) \\
\text { Lung diseases: } 8 / 30(26.67) \\
\text { Urinary diseases: } 3 / 30(10) \\
\text { Caroli disease: } 1 / 30(3.33) \\
\text { Liver cirrhosis: } 10 / 30(33.33) \\
\text { IgA deficiency: } 1 / 30(3.33) \\
\text { No associated conditions: } 1 / 30(3.33) \\
\text { Acute pancreatitis: } 1 / 30(3.33) \\
\text { Heart diseases: } 4 / 30(13.33)\end{array}$ \\
\hline Donor & $\begin{array}{l}1^{\text {st }} \text { degree relative: } 16 / 30(53.33) \\
2^{\text {nd }} \text { degree relative: } 4 / 30(13.33) \\
\text { wife: } 8 / 30(26.67) \\
\text { husband: } 2 / 30(6.67)\end{array}$ \\
\hline Relapse & $2 / 30(6.67)$ \\
\hline $\begin{array}{l}\text { Antibiotics administered } \\
\text { pre transplantation for other conditions }\end{array}$ & $\begin{array}{l}\text { Amoxicillin: } 5 / 29(17.24) \\
\text { Amoxicillin-clavulanate: 4/29 (13.79) } \\
\text { Trimetoprim-Sulfamethoxazole: } 2 / 29(6.9) \\
\text { Ceftriaxone: } 9 / 29(31.03) \\
\text { Ciprofloxacin: } 5 / 29(17.24) \\
\text { Colistin: } 2 / 29(6.9) \\
\text { Meropenem: } 1 / 29(3.45) \\
\text { Imipenem-cilastatin: } 1 / 29(3.45)\end{array}$ \\
\hline
\end{tabular}

Table III. Mean number (standard deviation) of relapses, according to antibiotherapy for Clostridium difficile.

\begin{tabular}{|c|c|c|c|c|}
\hline Antibiotherapy & metronidazol $(\mathrm{n}=5)$ & vancomicin $(n=4)$ & $\begin{array}{c}\text { vancomicin and } \\
\text { metronidazol }(n=21)\end{array}$ & P-value \\
\hline Number relapses, mean (SD) & $1(0)$ & $1.5(0.58)$ & $2.33(0.86)$ & 0.003 \\
\hline
\end{tabular}


Table IV. Clinical characteristics post transplantation

\begin{tabular}{|c|c|}
\hline $\begin{array}{l}\text { Nr bowel movements } \\
\text { at } 7 \text { days after trans- } \\
\text { plantation }\end{array}$ & $\begin{array}{l}1: 9 / 30(30) \\
2: 12 / 30(40) \\
3: 8 / 30(26.67) \\
4: 1 / 30(3.33)\end{array}$ \\
\hline $\begin{array}{l}\text { Fever after transplan- } \\
\text { tation }\end{array}$ & $8 / 30(26.67)$ \\
\hline $\begin{array}{l}\text { Abdominal pain after } \\
\text { transplantation }\end{array}$ & $\begin{array}{l}\text { no: } 4 / 30(13.33) \\
\text { yes: } 1 / 30(3.33) \\
\text { mild: } 13 / 30(43.33) \\
\text { moderate: } 10 / 30(33.33) \\
\text { severe: } 2 / 30(6.67)\end{array}$ \\
\hline $\begin{array}{l}\text { Nr bowel movements } \\
\text { at } 6 \text { months }\end{array}$ & $\begin{array}{l}1: 17 / 28(60.71) \\
2: 6 / 28(21.43) \\
3: 5 / 28(17.86)\end{array}$ \\
\hline $\begin{array}{l}\text { Nr bowel movements } \\
\text { at } 1 \text { year }\end{array}$ & $\begin{array}{l}1: 14 / 28(50) \\
2: 12 / 28(42.86) \\
3: 2 / 28(7.14)\end{array}$ \\
\hline
\end{tabular}

provement in the mean number of bowel movements at 7 days/ 6 months/ 1 year $(\mathrm{p}<0.001)$ (tabel V).

The choice of donors was found to be important in all recipients due to personal safety, even if there is no statistical significance $(\mathrm{p}=0.43)$ for the relationship between relapse and donor (the " $1^{\text {st }}$ and $2^{\text {nd }}$ degree relative" group) in our population. Sixteen patients chose a ${ }^{\text {st }}$ degree relative donor, 8 patients chose their wife, 4 patients a $2^{\text {nd }}$ degree relative and 2 patients, their husband.

We found a statistically significant relation between type of donor and number of bowel movements only at 6 months post FMT ( $\mathrm{p}=$ $0.01)$, but not at 1 year $(\mathrm{p}=0.82)$ (table VI).

Table V Number of bowel movements at different times (before transplantation, 7 day after transplantation, at 6 months and at 1 year)

\begin{tabular}{lcccc}
\hline Time: & before & 7 days & Difference $(95 \%$ CI) & P-value \\
Number of bowel movements, median (IQR) & $7(6-10)$ & $2(1-3)$ & $-5(-6.5--4.5)$ & $<0.001$ \\
\hline Time: & before & 6 months & & \\
Number of bowel movements, median (IQR) & $7(5.75-10)$ & $1(1-2)$ & $-6(-7--5)$ & $<0.001$ \\
\hline Time: & before & 1 year & & \\
Number of bowel movements, median (IQR) & $7(5.75-10)$ & $1.5(1-2)$ & $-5.5(-7--4.5)$ & $<0.001$ \\
\hline Time: & 7 days & 6 months & & 0.017 \\
Number of bowel movements, median (IQR) & $2(1-3)$ & $1(1-2)$ & $-1(-1.5--0.5)$ & 1 \\
\hline Time: & 6 months & 1 year & & \\
Number of bowel movements, median (IQR) & $1(1-2)$ & $1.5(1-2)$ & $0.5(-1-1)$ &
\end{tabular}

$\mathrm{IQR}$ - interquartile range; $\mathrm{CI}$ - confidence interval

Table VI. Median number ( $1^{\text {st }}$ quartile $-3^{\text {rd }}$ quartile) of bowel movements/day after transplantation (at 7 days, 6 months and 1 year), according to type of donor

\begin{tabular}{lccccc}
\hline Donor: & $\begin{array}{c}1^{\text {st }} \text { degree relative } \\
(\mathrm{n}=16)\end{array}$ & $\begin{array}{c}2^{\text {nd }} \text { degree } \\
\text { relative }(\mathrm{n}=4)\end{array}$ & $\begin{array}{c}\text { Wife } \\
(\mathrm{n}=8)\end{array}$ & $\begin{array}{c}\text { Husband } \\
(\mathrm{n}=2)\end{array}$ & P-value \\
\hline $\begin{array}{l}\text { Number of bowel movements/day, } \\
\text { at 7 days }\end{array}$ & $2(1.75-3)$ & $1(1-1.25)$ & $\begin{array}{c}2(1.75 \\
-2.25)\end{array}$ & $3(3-3)$ & 0.075 \\
\hline $\begin{array}{l}\text { Number of bowel movements/day } \\
\text { at 6 months }\end{array}$ & $1(1-1)$ & $3(2-3)$ & $1.5(1-2)$ & $\begin{array}{c}2.5(2.25- \\
2.75)\end{array}$ & 0.04 \\
\hline $\begin{array}{l}\text { Number of bowel movements/day } \\
\text { at 1 year }\end{array}$ & $1(1-2)$ & $1(1-1.5)$ & $2(1-2)$ & $\begin{array}{r}2(1.5- \\
2.5)\end{array}$ & 0.828 \\
\hline
\end{tabular}




\section{Discussion}

This 30-case series, the first prospective study published in Romania, of relapsing CDI patients treated with FMT using colonoscopy, provides effectiveness of the procedure for this condition.

Even though the literature generally recommends FMT from the third recurrence [13], in this study we are trying to track the evolution of patients after FMT since the first relapse.

Although there is a limited number of patients in this study, the high success rate of FMT in recurrent CDI has been observed (2 relapses out of the total of 30), as literature suggests $[13,14]$. The post-FMT outcome in 28 patients was favourable (had no toxin positive strains and no symptoms in the following 12 months).

As for the laboratory tests, all patients had a mild increase in inflammatory markers with respect to their initial levels (ESR and CRP) in the first week after FMT, but with subsequent return within normal range, as described in the literature [14]. Even though it did not show any impact on our study and there was a small number of patients, we mentioned this change in laboratory samples as it is also described in the literature.

Approximately $20 \%$ of patients infected with Clostridium difficile treated with antibiotics will experience a relapse within the first 6 months from discontinuation of the treatment [15]. The treatment options for relapsing Clostridium difficile are limited. ACG (The American College of Gastroenterology) recommends FMT after the $3^{\text {rd }}$ relapse $[16,17,18]$ in order to re-establish the normal composition of the intestinal flora, restore the metabolic balance and stimulate both cellular and humoral immune responses in the intestinal mucosa. The fecal matter transplantation has been used ever since the $4^{\text {th }}$ century in China, and it is increasingly accepted nowadays as a safe and effective method for the treatment of relapsing Clostridium difficile infections.
Clinical research is increasingly supplying information on the role of the intestinal microbiota in chronic diseases, such as IBD (Crohn's disease and ulcerative colitis), metabolic syndrome, various cancers and obesity [19].

In order to avoid adverse events, a vital stage in the preparation of FMT is the donor screening, which should include detailed laboratory tests. It is of utmost importance to ensure that the donors are healthy, without evidence of autoimmune diseases or other chronic conditions. Certain adverse events related to the FMT treatment have, however, been reported. Brandt and colleagues reported that in the long term, the 77 patients they were following up did not experience any infection post colonoscopy FMT, but that 4 patients later presented with new diseases - peripheral neuropathy, Sjogren's syndrome, idiopathic thrombocytopenic purpura and rheumatoid arthritis [20]. Others experienced improvement of pre-existent conditions, such as allergies, sinusitis and arthritis. These new diseases, as well as improvements of old diseases, cannot be directly linked to the FMT, but incite to further research on the interaction of the microbiota and autoimmune diseases [11,19-21].

The infusion of stool via colonoscopy has several advantages over other methods (enema, nasogastric tube, gastroscopy). Firstly, this method ensures infusion of stool on the entire length of the colon. Secondly, the colonic mucosa can be visualised directly and any abnormal aspects can be documented. Thirdly, the patients are sedated and generally tolerate the FMT well. In addition, the success rates range from $86 \%$ to $100 \%$, while the success rate for enema range from $81 \%$ to $100 \%$ [22]. Nevertheless, FMT via colonoscopy may incur risks of perforation, infection, bleeding and pain. Although the nasogastric method is less efficient, with rates of success between $73 \%$ and $83 \%$, it is easier to perform, less expensive, and has lower risks for the patient $[23,24]$. In our case, colonoscopy was 
considered more effective even if it presents certain risks. It was possible to visualize the entire colon mucosa and we could spread the prepared solution to the right side of the colon for better effectiveness. The "preferred donor" is usually someone sharing an intimate environment with the patient $[23,25]$.

Considering that each method of infusion has its advantages and disadvantages, the best method should be individualised for each patient.

We are aware of the limitations of our study: the limited number of subjects, the lack of a control group. Despite the strong belief of some practicing physicians in the effectiveness and safety of FMT, a randomised controlled trial may be necessary before this treatment is approved in the medical community. The standard donor, the optimal screening and treatment protocols should also be developed and validated

\section{Conclusion}

FMT for recurrent Clostridium difficile infections can be an effective and durable method of treatment for patients resistant to traditional treatment.

Transplantation of faecal suspension obtained from healthy donors may restore normal microbiota, breaking the cycle of recurrent CDI, usually after traditional treatment.

In most cases (28 patients), symptoms improved at 7 days of FMT procedure and patients stayed diarrhoea free for 12 months (our followup), indicating that FMT could be an effective alternative in the treatment of patients with recurrent/refractory CDI.

The only question that remains related to FMT is what will be the side effects over time?

As the Clostridium difficile "epidemics" show, an increasing number of patients with recurrent or refractory infections will be able to benefit from this method of treatment.

Despite not having any element of novelty, our study aims to provide yet another argument in favour of fecal microbiota transplantation and its importance and necessity in the treatment of recurrent Clostridium difficile infection.

Our study, the first prospective study of its kind in Romania, demonstrates the availability, simplicity and immediate and long-term effectiveness of FMT.

\section{Conflict-of-interes statement}

We declare that we have no conflicts of interes.

\section{References}

1. Bartlett JG. Clinical practice. Antibiotic associated diarrhea. N Engl J Med. 2002 Jan;346(5):334-9. DOI: 10.1056/NEJMcp011603

2. McFarland LV. Renewed interest in a difficult disease: Clostridium difficile infections - epidemiology and current treatment strategies. Curr Opin Gastroenterol. 2009 Jan;25(1):24-35. DOI: 10.1097/MOG.0b013e$32831 \mathrm{da} 7 \mathrm{c} 4$

3. Epuran S, Rusu E, Sbarcea A, Musat S. Clostridium difficile - etiology and epidemiology. Revista romana de boli infectioase 2014;1:28-30

4. Bauer MP, Notermans DW, van Benthem BH, Brazier JS, Wilcox MH, Rupnik M, et al. Clostridium difficile infection in Europe: a hospital based survey. Lancet. 2011 Jan;377(9759):63-73. DOI: 10.1016/S01406736(10)61266-4

5. Lee YC, Wang JT, Chen AC, Sheng WH, Chang SC, Chen YC. Changing incidence and clinical manifestations of Clostridium difficile-associated diarrhea detected by combination of glutamate dehydrogenase and toxin assay in Northern Taiwan. J Microbiol Immunol Infect. 2012 Aug;45(4):287-95. DOI: 10.1016/j. 
jmii.2011.12.001

6. Ray A, Smith R, Breaux J. Fecal microbiota transplantation for Clostridium Difficile infection: The Ochsner Experience. Ochsner J. 2014 Winter; 14(4): 538-44.

7. Bakken JS. Fecal bacteriotherapy for recurrent Clostridium difficile infection. Anaerobe 2009; 15:285. DOI: 10.1016/j.anaerobe.2009.09.007

8. Rubin TA, Gessert CE, Aas J, Bakken JS. Fecal microbiome transplantation for recurrent Clostridium difficile infection: report on a case series. Anaerobe. 2013 Feb;19:22-6. DOI: 10.1016/j.anaerobe.2012.11.004

9. Grehan MJ, Borody TJ, Leis SM, Campbell J, Mitchell $\mathrm{H}$, Wettstein A. Durable alteration of the colonic microbiota by the administration of donor fecal flora. J Clin Gastroenterol. 2010 Sep;44(8):551-61. DOI: 10.1097/ MCG.0b013e3181e5d06b

10. Brandt LJ, Reddy SS. Fecal microbiota transplantation for recurrent Clostridium difficile infection. J Clin Gastroenterol. 2011 Nov;45 Suppl:S159-67. DOI: 10.1097/ MCG.0b013e318222e603

11. Bang BW, Park JS, Kim HK, Shin YW, Kwon KS, Kwon HY, et al. Fecal Microbiota Transplantation for Refractory and Recurrent Clostridium difficile Infection: A Case Series of Nine Patients. Korean J Gastroenterol. 2017 Apr 25;69(4):226-31. DOI: 10.4166/ kjg.2017.69.4.226

12. Gough E, Shaikh H, Manges AR. Systematic review of intestinal microbiota transplantation (fecal bacteriotherapy) for recurrent Clostridium difficile infection. Clin Infect Dis. 2011 Nov;53(10):994-1002. DOI: $10.1093 / \mathrm{cid} / \mathrm{cir} 632$

13. Michael B. Edmond. The power of poop: Fecal microbiota transplantation for Clostridium Difficile infection. Trans Am Clin Climatol Assoc. 2016; 127: 71-80.

14. Konturek PC, Koziel J, Dieterich W, et al. Successful therapy of Clostridium difficile infection with fecal microbiota transplantation. J Physiol Pharmacol. 2016 Dec;67(6):859-66.

15. Eyre DW, Walker AS, Wyllie D, Dingle KE, Griffiths D, Finney J, et al. Predictors of First Recurrence of Clostridium difficile Infection: Implications for Initial Management. Clin Infect Dis. 2012 Aug; 55(2): S77S87. DOI: $10.1093 / \mathrm{cid} / \mathrm{cis} 356$

16. Surawicz CM, Brandt LJ, Binion DG, Ananthakrishnan AN, Curry SR, Gilligan PH, et al. Guidelines for diag- nosis, treatment, and prevention of Clostridium difficile infections. Am J Gastroenterol. 2013 Apr;108(4):47898. DOI: 10.1038/ajg.2013.4

17. Boyle ML, Ruth-Sahd LA, Zhou Z. Fecal Microbiota Transplant to Treat Recurrent Clostridium difficile Infections. Crit Care Nurse. 2015 Apr;35(2):51-64. DOI: $10.4037 / \operatorname{ccn} 2015356$

18. Guo B, Harstall C, Louie T, Veldhuyzen van Zanten S, Dieleman LA. Systematic review: faecal transplantation for the treatment of Clostridium difficile-associated disease. Aliment Pharmacol Ther. 2012 Apr;35(8):86575. DOI: 10.1111/j.1365-2036.2012.05033.x

19. Cohen SH, Gerding DN, Johnson S, Kelly CP, Loo VG, McDonald LC, et al. Clinical practice guidelines for Clostridium difficile infection in adults: 2010 update by the Society for Health Epidemiology of America (SHEA) and the Infectious Diseases Society of America. Infect Control Hosp Epidemiol. 2010 May;31(5):431-55. DOI: 10.1086/651706

20. Festi D, Schiumerini R, Birtolo C, Marzi L, Montrone L, Scaioli E, et al. Gut microbiota and its pathophysiology in disease paradigms. Dig Dis. 2011;29(6):518-24. DOI: $10.1159 / 000332975$

21. Brandt LJ, Aroniadis OC, Mellow M, Kanatzar A, Kelly C, Park T, et al. Long-term follow-up of colonoscopic fecal microbiota transplant for recurrent Clostridium difficile infection. Am J Gastroenterol. 2012 Jul;107(7):1079-87. DOI: 10.1038/ajg.2012.60

22. Mattila E, Uusitalo-Seppala R, Wuorela M, Lehtola L, Nurmi H, Ristikankare $\mathrm{M}$ et al. Fecal transplantation, through colonoscopy, is effective therapy for recurrent Clostridium difficile infection. Gastroenterology. 2012 Mar;142(3):490-6. DOI: 10.1053/j.gastro.2011.11.037

23. Aas J, Gessert CE, Bakken JS. Recurrent Clostridium difficile colitis: aseries involving 18 patients treated with donor stool administered via a nasogastric tube. Clin Infect Dis. 2003 Mar;36(5):580-5. DOI: $10.1086 / 367657$

24. Rao K, Safdar N. Fecal microbiota for the treatment of Clostridium Difficile infection. J Hosp Med. 2016 Jan;11(1):56-61. DOI: 10.1002/jhm.2449

25. Gupta A, Khanna S. Fecal microbiota transplantation. JAMA. 2017 July;318(1):102. DOI: 10.1001/ jama.2017.6466 